\title{
The New Viscosity Approximation Methods for Nonexpansive Nonself-Mappings
}

\author{
Chao Liu, Meimei Song \\ College of Science, Tianjin University of Finance and Economics Pearl River College, Tianjin, \\ China \\ Email: liuchao2071@163.com, songmeimei@tjut.edu.cn
}

Received 30 April 2016; accepted 27 June 2016; published 30 June 2016

Copyright @ 2016 by authors and Scientific Research Publishing Inc.

This work is licensed under the Creative Commons Attribution International License (CC BY). http://creativecommons.org/licenses/by/4.0/

(c) (i) Open Access

\section{Abstract}

In this paper, to find the fixed points of the nonexpansive nonself-mappings, we introduced two new viscosity approximation methods, and then we prove the iterative sequences defined by above viscosity approximation methods which converge strongly to the fixed points of nonexpansive nonself-mappings. The results presented in this paper extend and improve the results of Song-Chen [1] and Song-Li [2].

\section{Keywords}

Fixed Points, Nonexpansive Nonself-Mappings, Viscosity Approximation Methods, Real Banach Space

\section{Introduction}

Let $C$ be a closed convex subset of a Hilbert space $H$ and $T: C \rightarrow C$ a nonexpansive mapping (i.e., $\|T x-T y\| \leq\|x-y\|$ for any $x, y \in C)$. Let $u \in C$ be a fixed point of $T$. Then for any initial $x_{0} \in C$ and real sequence $\left\{\lambda_{n}\right\} \subset(0,1)$, we define a sequence $\left\{x_{n}\right\}$ by

$$
x_{n+1}=\lambda_{n+1} u+\left(1-\lambda_{n+1}\right) T x_{n}(n \geq 0)
$$

Helpern [3] was the first to study the strong convergence of the iteration process (1). In 1992, Albert [4] studied the convergence of the Ishikawa iteration process in Banach space, which was extended the results of Mann iteration process [5]. But the mappings in these results must be self-mapping and continuous. It is more useful to get some results for nonself-mappings.

In 2006, Yisheng Song and Rudong Chen [1] studied viscosity approximation methods for nonexpansive nonself-mappings by the following iterative sequence $\left\{x_{n}\right\}$. 


$$
x_{n+1}=P\left(\alpha_{n} f\left(x_{n}\right)+\left(1-\alpha_{n}\right) T x_{n}\right) \quad\left(0<\alpha_{n}<1\right)
$$

where $X$ is a real reflexive Banach space, and $C$ is a closed subset of $X$ which is also a sunny nonexpansive retract of $X . T: C \rightarrow X$ is a nonexpansive mapping, $f: C \rightarrow C$ is a fixed contractive mapping and $P$ is a sunny nonexpansive retraction of $X$ onto $C$.

In 2007, Yisheng Song and Qingchun Li [2] found a new viscosity approximation method for nonexpansive nonself-mappings as follows

$$
x_{n+1}=\alpha_{n} f\left(x_{n}\right)+\left(1-\alpha_{n}\right) P T x_{n} \quad\left(0<\alpha_{n}<1\right)
$$

where $X$ is a real reflexive Banach space, and $C$ is a closed subset of $X$ which is also a sunny nonexpansive retract of $X . \quad T: C \rightarrow X$ is a nonexpansive mapping, $f: C \rightarrow C$ is a fixed contractive mapping and $P$ is a sunny nonexpansive retraction of $X$ onto $C$.

In this paper, we will study two new viscosity approximation methods for nonexpansive nonself-mappings in reflexive Banach space $X$, which can extend the results of Song-Chen [1] and Song-Li [2] on the twodimensional space.

Let us start by making some basic definitions.

\section{Preliminary Notes}

Let $X$ be a real Banach space with the norm $\|\cdot\|$, and $X^{*}$ be its dual space. When $\left\{x_{n}\right\}$ is a sequence in $X$, the $x_{n} \rightarrow x \quad$ (respectively $x_{n} \stackrel{w}{\longrightarrow} x, x_{n} \stackrel{w^{*}}{\longrightarrow} x$ ) will denote the strong (respectively the weak, the weak star) convergence of the sequence $x_{n}$ to $x$.

Definition 2.1. Let $X$ be a real Banach space and $J$ denote the normalized duality mapping from $X$ into $2^{E^{*}}$ given by

$$
J(X)=\left\{f \in E^{*}:\langle x, f\rangle=\|x\|\|f\|\|,\| x\|=\| f \|\right\} \text { for all } x \in X,
$$

where $X^{*}$ denotes the dual space of $X$ and $\langle\cdot, \cdot\rangle$ denotes the generalized duality pairing.

Let $F(T)$ denotes set of the fixed point of $T$.

Definition 2.2. Let $X$ ba a real Banach space and $T$ a mapping with domain $D(T)$ and range $R(T)$ in T.T is called nonexpansive if for any $x, y \in D(T)$, such that $\|T x-T y\| \leq\|x-y\|$ (respectively $T$ is called contractive if for any $x, y \in D(T)$, such that $\|T x-T y\| \leq \beta\|x-y\|)$, where $0<\beta<1$.

Definition 2.3. Let $X$ be a Banach space, $C$ and $D$ be nonempty subsets of $X, D \subset C$. A mapping $P: C \rightarrow D$ is called a retraction from $C$ to $D$, if $P$ is continuous with $F(P)=D$. A mapping $P: C \rightarrow D$ is called a sunny, if $P(P x+t(x-P x))=P x$, for all $x \in C, t>0$, whenever $P x+t(x-P x) \in C$. And a subset $D$ of $C$ is said to be a sunny nonexpansive retract of $C$, if there exists a sunny nonexpansive retraction of $C$ onto $D$.

Definition 2.4. Let $X$ be a real reflexive Banach space, which admits a weakly sequentially continuous duality mapping from $X$ to $X^{*}$, and $C$ be a closed convex subset of $X$, which is also a sunny nonexpansive retract of $X$, and $T: C \rightarrow X$ be nonexpansive mapping satisfying the weakly inward condition and $F(T) \neq \phi$, and $f: C \rightarrow C$ is called contractive mapping. For a given $x_{0} \in C$ and $n \in \mathrm{N}$, let us define $\left\{x_{n}\right\}$ and $\left\{y_{n}\right\}$ by the following iterative scheme:

$$
\left\{\begin{array}{l}
x_{n+1}=P\left(\alpha_{n} f\left(x_{n}\right)+\left(1-\alpha_{n}\right) T y_{n}\right) \\
y_{n}=\beta_{n} x_{n}+\left(1-\beta_{n}\right) T x_{n}
\end{array}\right.
$$

where $\alpha_{n}, \beta_{n} \in(0,1), \lim _{n \rightarrow \infty} \alpha_{n}=0, \lim _{n \rightarrow \infty} \beta_{n}=1$.

$$
\left\{\begin{array}{l}
x_{n+1}=\alpha_{n} f\left(x_{n}\right)+\left(1-\alpha_{n}\right) P T y_{n} \\
y_{n}=\beta_{n} x_{n}+\left(1-\beta_{n}\right) T x_{n}
\end{array}\right.
$$

where $\alpha_{n}, \beta_{n} \in(0,1), \lim \alpha_{n}=0, \lim \beta_{n}=1$.

We call (2) the first type viscosity approximation method for nonexpansive nonself-mapping and call (3) the second type viscosity approximation method for nonexpansive nonself-mapping. 
Let us introduce some lemmas, which play important roles in our results.

Lemma 2.1. ([6]) Let $X$ be a real Banachspace, then for each $x, y \in X$, the following inequality holds:

$$
\|x+y\|^{2} \leq\|x\|^{2}+2\langle y, j(x+y)\rangle \text {, for } j(x+y) \in J(x+y)
$$

Lemma 2.2. ([7]) Let $\left\{a_{n}\right\},\left\{b_{n}\right\},\left\{c_{n}\right\}$ be three nonnegative real sequences satisfying

$$
a_{n+1} \leq\left(1-t_{n}\right) a_{n}+b_{n}+c_{n} \text { with }\left\{t_{n}\right\} \subset[0,1], \sum_{n=0}^{\infty} c_{n}<\infty .
$$

Then $a_{n} \rightarrow 0$ as $n \rightarrow \infty$.

Lemma 2.3. ([1]) Let $X$ be a real smooth Banach space, and $C$ be nonempty closed convex subset of $X$, which is also a sunny nonexpansive retract of $X$ and $T: C \rightarrow X$ be mapping satisfying the weakly inward condition, and $P$ be a sunny nonexpansive retraction of $X$ onto $C$, then $F(T)=F(P T)$.

Lemma 2.4. ([1]) Let $C$ be nonempty closed convex subset of a reflexive Banach space $X$ which satisfies Opial's condition, and suppose $T: C \rightarrow X$ is nonexpansive. Then the mapping $I-T$ is demiclosed at zero, i.e., $x_{n} \stackrel{w}{\longrightarrow} x, x_{n}-T x_{n} \rightarrow 0$ implies $x=T x$.

\section{Main Results}

First of all, let us study the first type viscosity approximation for nonexpansive nonself-mappings.

Lemma 3.1. ([1]) Let $X$ be a reflexive Banach space which admits a weakly sequentially continuous duality mapping $J$ from $X$ to $X^{*}$. Suppose $C$ is a nonexpansive retract of $X$ which is also a sunny nonexpansive retract of $X$, and $T: C \rightarrow X$ is a nonexpansive mapping satisfying the weakly inward condition and $F(T) \neq \phi$, let $f: C \rightarrow C$ be a fixed contractive mapping from $C$ to $C$. Let $x_{t} \in C$ be the unique fixed point of $T$, that is,

$$
x_{t}=P\left(t f\left(x_{t}\right)+(1-t) T x_{t}\right) \text {, for any } t \in(0,1) \text {, }
$$

where $P$ is a sunny nonexpansive retract of $X$ onto $C$. Then as $t \rightarrow 0,\left\{x_{t}\right\}$ converges strongly to some fixed point $p$ of $T$. And $p$ is the unique solution in $F(T)$ to the following variational inequality

$$
\langle(I-f) p, j(p-u)\rangle \leq 0
$$

For all $u \in F(T)$.

Lemma 3.2. Let $X$ be a reflexive Banach space which admits a weakly sequentially continuous duality mapping $J$ from $X$ to $X^{*}$. Suppose $C$ is a nonexpansive retract of $X$, which is also a sunny nonexpansive retract of $X$ and $T: C \rightarrow X$ is a nonexpansive mapping satisfying the weakly inward condition and $F(T) \neq \phi$, let $f: C \rightarrow C$ be a fixed contractive mapping from $C$ to $C$. And $\left\{x_{n}\right\}_{n=0}^{\infty}$ is a sequence by definition $2.4(2)$, then the sequence $\left\{x_{n}\right\}$ is bounded.

Proof. Let $p \in F(T)$, so we have

$$
\begin{aligned}
\left\|x_{n+1}-p\right\| & =\left\|P\left(\alpha_{n} f\left(x_{n}\right)+\left(1-\alpha_{n}\right) T y_{n}\right)-p\right\| \\
& \leq\left\|\alpha_{n} f\left(x_{n}\right)+\left(1-\alpha_{n}\right) T y_{n}-p\right\| \\
& =\alpha_{n}\left\|f\left(x_{n}\right)-f(p)\right\|+\left(1-\alpha_{n}\right)\left\|T y_{n}-p\right\|+\alpha_{n}\|f(p)-p\| \\
& \leq \alpha_{n}\left\|f\left(x_{n}\right)-f(p)\right\|+\left(1-\alpha_{n}\right)\left\|y_{n}-p\right\|+\alpha_{n}\|f(p)-p\| \\
& \leq \alpha_{n} \beta\left\|x_{n}-p\right\|+\left(1-\alpha_{n}\right)\left\|y_{n}-p\right\|+\|f(p)-p\|
\end{aligned}
$$

while,

$$
\begin{aligned}
\left\|y_{n}-p\right\| & =\left\|\beta_{n} x_{n}+\left(1-\beta_{n}\right) T x_{n}-p\right\| \\
& \leq\left\|\beta_{n}\left(x_{n}-p\right)+\left(1-\beta_{n}\right)\left(T x_{n}-p\right)\right\| \\
& \leq \beta_{n}\left\|x_{n}-p\right\|+\left(1-\beta_{n}\right)\left\|T x_{n}-p\right\| \\
& \leq\left\|x_{n}-p\right\|
\end{aligned}
$$


therefore,

$$
\begin{aligned}
\left\|x_{n+1}-p\right\| & \leq \alpha_{n} \beta\left\|x_{n}-p\right\|+\left(1-\alpha_{n}\right)\left\|x_{n}-p\right\|+\|f(p)-p\| \\
& =\left(1-\alpha_{n}+\alpha_{n} \beta\right)\left\|x_{n}-p\right\|+\|f(p)-p\| \\
& =\left(1-\alpha_{n}(1-\beta)\right)\left\|x_{n}-p\right\|+\|f(p)-p\|
\end{aligned}
$$

since $\left(1-\alpha_{n}(1-\beta)\right) \in(0,1)$,

therefore $\left\|x_{n+1}-p\right\| \leq \max \left\{\left\|x_{0}-p\right\|,\|f(p)-p\|\right\}$, then $\left\{x_{n}\right\}$ is bounded.

Lemma 3.3. Let $X$ be a reflexive Banach space which admits a weakly sequentially continuous duality mapping $J$ from $X$ to $X^{*}$. Suppose $C$ is a nonexpansive retract of $X$ which is also a sunny nonexpansive retract of $X$, and $T: C \rightarrow X$ is a nonexpansive mapping satisfying the weakly inward condition and $F(T) \neq \phi$, let $f: C \rightarrow C$ be a fixed contractive mapping from $C$ to $C$. And $\left\{x_{n}\right\}_{n=0}^{\infty}$ is a sequence by definition 2.4 (2). Let us assume that there are two sequences $\left\{\alpha_{n}\right\},\left\{\beta_{n}\right\}$ in $[0,1]$ satisfying the following conditions:

$$
\sum_{n=1}^{\infty}\left(\left|\alpha_{n}-\alpha_{n-1}\right|+\left|\beta_{n}-\beta_{n-1}\right|\right)<\infty
$$

then

1) $\lim _{n \rightarrow \infty}\left\|x_{n}-x_{n+1}\right\|=0$

2) $\lim _{n \rightarrow \infty}\left\|x_{n}-P T x_{n}\right\|=0$

Proof by lemma 3.2, we know that the sequence $\left\{x_{n}\right\}$ is bounded. So the sequences $\left\{f\left(x_{n}\right)\right\},\left\{y_{n}\right\}, T x_{n}$ are also bounded. Therefore, we have

$$
\begin{gathered}
\left\|y_{n}-y_{n-1}\right\|=\left\|\beta_{n} x_{n}+\left(1-\beta_{n}\right) T x_{n}-\beta_{n-1} x_{n-1}-\left(1-\beta_{n-1}\right) T x_{n-1}\right\| \\
\quad=\left\|\beta_{n}\left(x_{n}-x_{n-1}\right)+\left(1-\beta_{n}\right)\left(T x_{n}-T x_{n-1}\right)+\left(\beta_{n}-\beta_{n-1}\right)\left(x_{n-1}-T x_{n-1}\right)\right\| \\
\quad \leq \beta_{n}\left\|x_{n}-x_{n-1}\right\|+\left(1-\beta_{n}\right)\left\|T x_{n}-T x_{n-1}\right\|+\mid \beta_{n}-\beta_{n-1}\left\|x_{n-1}-T x_{n-1}\right\| \\
\quad \leq\left\|x_{n}-x_{n-1}\right\|+\mid \beta_{n-1}-\beta_{n}\left\|x_{n-1}-T x_{n-1}\right\| \\
\begin{aligned}
\left\|x_{n+1}-x_{n}\right\|= & \| P\left(\alpha_{n} f\left(x_{n}\right)+\left(1-\alpha_{n}\right) T y_{n}\right)-P\left(\alpha_{n-1} f\left(x_{n-1}\right)+\left(\left(1-\alpha_{n-1}\right) T y_{n-1}\right) \|\right. \\
\leq & \left\|\alpha_{n} f\left(x_{n}\right)+\left(1-\alpha_{n}\right) T y_{n}-\alpha_{n-1} f\left(x_{n-1}\right)-\left(1-\alpha_{n-1}\right) T y_{n-1}\right\| \\
= & \left\|\alpha_{n}\left(f\left(x_{n}\right)-f\left(x_{n-1}\right)\right)+\left(\alpha_{n}-\alpha_{n-1}\right)\left(f\left(x_{n-1}\right)-T y_{n-1}\right)+\left(1-\alpha_{n}\right)\left(T y_{n}-T y_{n-1}\right)\right\| \\
= & \alpha_{n} \beta\left\|x_{n}-x_{n-1}\right\|+\left|\alpha_{n}-\alpha_{n-1}\right|\left\|f\left(x_{n-1}\right)-T y_{n-1}\right\|+\left(1-\alpha_{n}\right)\left\|y_{n}-y_{n-1}\right\|
\end{aligned}
\end{gathered}
$$

by (4), we have

$$
\begin{aligned}
\left\|x_{n+1}-x_{n}\right\| \leq & \alpha_{n} \beta\left\|x_{n}-x_{n-1}\right\|+\left|\alpha_{n}-\alpha_{n-1}\right|\left\|f\left(x_{n-1}\right)-T y_{n-1}\right\| \\
& +\left(1-\alpha_{n}\right)\left(\left\|x_{n}-x_{n-1}\right\|+\left|\beta_{n}-\beta_{n-1}\right|\left\|x_{n-1}-T x_{n-1}\right\|\right) \\
\leq & \alpha_{n} \beta\left\|x_{n}-x_{n-1}\right\|+\left|\alpha_{n}-\alpha_{n-1}\right|\left\|f\left(x_{n-1}\right)-T y_{n-1}\right\| \\
& +\left(1-\alpha_{n}\right)\left\|x_{n}-x_{n-1}\right\|+\left|\beta_{n}-\beta_{n-1}\right|\left\|x_{n-1}-T x_{n-1}\right\|
\end{aligned}
$$

Set $M_{1}=\max \left\{\left\|x_{n-1}-T x_{n-1}\right\|,\left\|f\left(x_{n-1}\right)-T y_{n-1}\right\|\right\}$

$$
\begin{aligned}
\left\|x_{n+1}-x_{n}\right\| & \leq\left(1-\alpha_{n}+\alpha_{n} \beta\right)\left\|x_{n}-x_{n-1}\right\|+\left(\left|\alpha_{n-1}-\alpha_{n}\right|+\left|\beta_{n-1}-\beta_{n}\right|\right) M_{1} \\
& \leq\left(1-\alpha_{n}(1-\beta)\right)\left\|x_{n}-x_{n-1}\right\|+\left(\left|\alpha_{n-1}-\alpha_{n}\right|+\left|\beta_{n-1}-\beta_{n}\right|\right) M_{1}
\end{aligned}
$$

Set $a_{n+1}=\left\|x_{n+1}-x_{n}\right\|, \quad t_{n}=1-\alpha_{n}(1-\beta), \quad b_{n}=0, \quad c_{n}=\left(\left|\alpha_{n-1}-\alpha_{n}\right|+\left|\beta_{n-1}-\beta_{n}\right|\right) M_{1}$ 
by the lemma 2.2 we have

$$
\lim _{n \rightarrow \infty}\left\|x_{n}-x_{n+1}\right\|=0
$$

Now we will proof $\left\|x_{n}-P T x_{n}\right\| \rightarrow 0$ as $n \rightarrow \infty$.

$$
\begin{aligned}
\left\|x_{n}-P T x_{n}\right\| & =\left\|x_{n}-x_{n+1}\right\|+\left\|x_{n+1}-P T x_{n}\right\| \\
& \leq\left\|x_{n}-x_{n+1}\right\|+\left\|P\left(\alpha_{n} f\left(x_{n}\right)+\left(1-\alpha_{n}\right) T x_{n}\right)-P T x_{n}\right\| \\
& \leq\left\|x_{n}-x_{n+1}\right\|+\left\|\alpha_{n} f\left(x_{n}\right)+\left(1-\alpha_{n}\right) T x_{n}-T x_{n}\right\| \\
& \leq\left\|x_{n}-x_{n+1}\right\|+\alpha_{n}\left\|f\left(x_{n}\right)-T x_{n}\right\|
\end{aligned}
$$

as $n \rightarrow \infty, \alpha_{n} \rightarrow 0$ therefore

$$
\lim _{n \rightarrow \infty}\left\|x_{n}-P T x_{n}\right\|=0 .
$$

Remark 3.1. From the lemma 3.1 we know that $p$ is the unique solution in $F(T)$ to the following variational inequality:

$$
\langle(I-F) p, j(p-u)\rangle \leq 0 \text { for all } u \in F(T) .
$$

Now, we can take a subsequence $\left\{x_{n_{k}}\right\}$ of $\left\{x_{n}\right\}$ such that

$$
\limsup _{n \rightarrow \infty}\left\langle f(p)-p, j\left(x_{n}-p\right)\right\rangle=\limsup _{k \rightarrow \infty}\left\langle f(p)-p, j\left(x_{n_{k}}-p\right)\right\rangle
$$

we may assume that $x_{n_{k}} \rightarrow x^{*}$ by $X$ is reflexive and $\left\{x_{n}\right\}$ is bounded. It follows from Lemma 2.3, Lemma 2.4, and (3.3), we have $x^{*} \in F(T)=F(P T)$, by (7) we have

$$
\limsup _{n \rightarrow \infty}\left\langle f(p)-p, j\left(x_{n}-p\right)\right\rangle=\limsup _{k \rightarrow \infty}\left\langle f(p)-p, j\left(x_{n_{k}}-p\right)\right\rangle \leq 0 .
$$

Theorem 3.4. Let $X$ be a reflexive Banach space which admits a weakly sequentially continuous duality mapping $J$ from $X$ to $X^{*}$. Suppose $C$ is a nonexpansive retract of $X$ which is also a sunny nonexpansive retract of $X$, and $T: C \rightarrow X$ is a nonexpansive mapping satisfying the weakly inward condition and $F(T) \neq \phi$, let $f: C \rightarrow C$ be a fixed contractive mapping from $C$ to $C$. And $\left\{x_{n}\right\}_{n=0}^{\infty}$ is the sequence by definition 2.4 (2). Let us assume there are two sequences $\left\{\alpha_{n}\right\},\left\{\beta_{n}\right\}$ in $[0,1]$ satisfying the following conditions:

$$
\sum_{n=1}^{\infty}\left(\left|\alpha_{n}-\alpha_{n-1}\right|+\left|\beta_{n}-\beta_{n-1}\right|\right)<\infty
$$

then the sequence $\left\{x_{n}\right\}$ converges strongly to the unique solution $p$ of the variational inequality:

$$
p \in F(T) \text { and }\langle(I-f) p, j(p-u)\rangle \leq 0 \text { for all } u \in F(T) .
$$

Proof. Since $C$ is closed, by lemma 3.2, $\left\{x_{n}\right\}$ is bounded, so $\left\{f\left(x_{n}\right)\right\},\left\{y_{n}\right\},\left\{T x_{n}\right\}$ are also bounded. Let $\left\{x_{t}\right\}$ be the sequence defined by

$$
x_{t}=P\left(t f\left(x_{t}\right)+(1-t) T x_{t}\right)
$$

by the lemma 3.1 as $t \rightarrow 0$ we have $\left\{x_{t}\right\}$ converges strongly to a fixed point $p$ of $T$ and $p$ is also the unique solution in $F(T)$ to the following variational inequality

$$
\langle(I-f) p, j(p-u)\rangle \leq 0 \text { for all } u \in F(T)
$$

using the remark 3.1, we have

$$
\limsup _{n \rightarrow \infty}\left\langle f(p)-p, j\left(x_{n}-p\right)\right\rangle \leq 0 .
$$


By the definition 2.4 (2), we have

$$
\begin{aligned}
\left\|x_{n+1}-p\right\|^{2}= & \left\|P\left(\alpha_{n} f\left(x_{n}\right)+\left(1-\alpha_{n}\right) T y_{n}\right)-p\right\|^{2} \\
\leq & \left\|\alpha_{n} f\left(x_{n}\right)+\left(1-\alpha_{n}\right) T y_{n}-p\right\|^{2} \\
\leq & \left\|\alpha_{n}\left(f\left(x_{n}\right)-p\right)+\left(1-\alpha_{n}\right)\left(T y_{n}-p\right)\right\|^{2} \\
\leq & \left(1-\alpha_{n}\right)^{2}\left\|y_{n}-p\right\|^{2}+2 \alpha_{n}\left\langle f\left(x_{n}\right)-f(p), j\left(x_{n+1}-p\right)\right\rangle \\
& +2 \alpha_{n}\left\langle f(p)-p, j\left(x_{n+1}-p\right)\right\rangle \\
\leq & \left(1-\alpha_{n}\right)^{2}\left\|y_{n}-p\right\|^{2}+2 \alpha_{n} \beta\left\|x_{n}-p\right\|\left\|x_{n+1}-p\right\| \\
& +2 \alpha_{n}\left\langle f(p)-p, j\left(x_{n+1}-p\right)\right\rangle \\
\leq & \left(1-\alpha_{n}\right)^{2}\left\|y_{n}-p\right\|^{2}+\alpha_{n} \beta\left(\left\|x_{n}-p\right\|^{2}+\left\|x_{n+1}-p\right\|^{2}\right) \\
& +2 \alpha_{n}\left\langle f(p)-p, j\left(x_{n+1}-p\right)\right\rangle
\end{aligned}
$$

While

$$
\begin{aligned}
\left\|y_{n}-p\right\| & =\left\|\beta_{n} x_{n}+\left(1-\beta_{n}\right) T x_{n}-p\right\| \leq \beta_{n}\left\|x_{n}-p\right\|+\left(1-\beta_{n}\right)\left\|T x_{n}-p\right\| \\
& \leq \beta_{n}\left\|x_{n}-p\right\|+\left(1-\beta_{n}\right)\left\|x_{n}-p\right\|=\left\|x_{n}-p\right\|
\end{aligned}
$$

therefore,

$$
\begin{aligned}
\left\|x_{n+1}-p\right\|^{2} & \leq \frac{\left(1-\alpha_{n}\right)^{2}+\alpha_{n} \beta}{1-\alpha_{n} \beta}\left\|x_{n}-p\right\|^{2}+\frac{2 \alpha_{n}}{1-\alpha_{n} \beta}\left\langle f(p)-p, j\left(x_{n+1}-p\right)\right\rangle \\
& \leq\left(1-\frac{1-2 \alpha_{n} \beta}{1-\alpha_{n} \beta}\right)\left\|x_{n}-p\right\|^{2}+\frac{\left(1-\alpha_{n}\right)^{2}}{1-\alpha_{n} \beta}\left\|x_{n}-p\right\|^{2}+\frac{2 \alpha_{n}}{1-\alpha_{n} \beta} r_{n+1}
\end{aligned}
$$

where $r_{n+1}=\max \left\{\left\langle f(p)-p, j\left(x_{n+1}-p\right)\right\rangle, 0\right\}$.

Setting $a_{n}=\left\|x_{n}-p\right\|, \quad t_{n}=1-\frac{1-2 \alpha_{n} \beta}{1-\alpha_{n} \beta}, \quad b_{n}=\frac{\left(1-\alpha_{n}\right)^{2}}{1-\alpha_{n} \beta}\left\|x_{n}-p\right\|^{2}, \quad c_{n}=\frac{2 \alpha_{n}}{1-\alpha_{n} \beta} r_{n+1}$ and applying Lemma

2.1, we conclude that $x_{n} \rightarrow p$.

Let us prove $p$ is the unique fixed point of $T$.

We assume that $p^{*}$ is another solution of (7) in $F(T)$, then $\left\langle f(p)-p, j\left(p^{*}-p\right)\right\rangle \leq 0$ and $\left\langle f\left(p^{*}\right)-p^{*}, j\left(p-p^{*}\right)\right\rangle \leq 0$, so we have $(1-\alpha)\left\|p-p^{*}\right\| \leq 0$, which implies the equality $p=p^{*}$.

Remark 3.2. when $\beta_{n}=1$ for all $n \in \mathrm{N}$. The first type viscosity approximation methods for nonexpansive nonself-mappings (see definition 2.4 ) become the following iteration sequence:

$$
x_{n+1}=P\left(\alpha_{n} f\left(x_{n}\right)+\left(1-\alpha_{n}\right) T x_{n}\right) .
$$

So the theorem 3.4 improves the theorem 2.4 of Song-Chen [1].

Now let us study the second type viscosity approximation for nonexpansive nonself-mappings.

Lemma 3.5. Let $X$ be a reflexive Banach space which admits a weakly sequentially continuous duality mapping $J$ from $X$ to $X^{*}$. Suppose $C$ is a nonexpansive retract of $X$, which is also a sunny nonexpansive retract of $X$ and $T: C \rightarrow X$ is a nonexpansive mapping satisfying the weakly inward condition and $F(T) \neq \phi$, let $f: C \rightarrow C$ be a fixed contractive mapping from $C$ to $C$. And $\left\{x_{n}\right\}_{n=0}^{\infty}$ is a sequence by definition 2.4 (3), then the sequence $\left\{x_{n}\right\}$ is bounded.

Proof. Let $p \in F(T)$, so we have 


$$
\begin{aligned}
\left\|x_{n+1}-p\right\| & =\left\|\alpha_{n} f\left(x_{n}\right)+\left(1-\alpha_{n}\right) P T y_{n}-p\right\| \\
& \leq\left\|\alpha_{n}\left(f\left(x_{n}\right)-f(p)\right)+\alpha_{n}(f(p)-p)+\left(1-\alpha_{n}\right)\left(P T y_{n}-p\right)\right\| \\
& \leq \alpha_{n}\left\|f\left(x_{n}\right)-f(p)\right\|+\left(1-\alpha_{n}\right)\left\|y_{n}-p\right\|+\alpha_{n}\|f(p)-p\| \\
& \leq \alpha_{n} \beta\left\|x_{n}-p\right\|+\left(1-\alpha_{n}\right)\left\|y_{n}-p\right\|+\alpha_{n}\|f(p)-p\|
\end{aligned}
$$

while,

$$
\begin{aligned}
\left\|y_{n}-p\right\| & =\left\|\beta_{n} x_{n}+\left(1-\beta_{n}\right) T x_{n}-p\right\| \\
& \leq\left\|\beta_{n}\left(x_{n}-p\right)+\left(1-\beta_{n}\right)\left(T x_{n}-p\right)\right\| \\
& \leq \beta_{n}\left\|x_{n}-p\right\|+\left(1-\beta_{n}\right)\left\|T x_{n}-p\right\| \\
& \leq\left\|x_{n}-p\right\|
\end{aligned}
$$

therefore,

$$
\begin{aligned}
\left\|x_{n+1}-p\right\| & \leq\left(1-\alpha_{n}+\alpha_{n} \beta\right)\left\|x_{n}-p\right\|+\|f(p)-p\| \\
& =\left(1-\alpha_{n}(1-\beta)\right)\left\|x_{n}-p\right\|+\|f(p)-p\|
\end{aligned}
$$

since $\left(1-\alpha_{n}(1-\beta)\right) \in(0,1)$,

therefore $\left\|x_{n+1}-p\right\| \leq \max \left\{\left\|x_{0}-p\right\|,\|f(p)-p\|\right\}$, then $\left\{x_{n}\right\}$ is bounded.

Lemma 3.6. ([2]) Let $X$ be a reflexive Banach space which admits a weakly sequentially continuous duality mapping $J$ from $X$ to $X^{*}$. Suppose $C$ is a nonexpansive retract of $X$ which is also a sunny nonexpansive retract of $X$, and $T: C \rightarrow X$ is a nonexpansive mapping satisfying the weakly inward condition and $F(T) \neq \phi$, let $f: C \rightarrow C$ be a fixed contractive mapping from $C$ to $C$. Let $x_{t} \in C$ be the unique fixed point of $T$, that is,

$$
x_{t}=t f\left(x_{t}\right)+(1-t) P T x_{t} \text {, for any } t \in(0,1),
$$

where $P$ is a sunny nonexpansive retract of $X$ onto $C$. Then as $t \rightarrow 0,\left\{x_{t}\right\}$ converges strongly to some fixed point $p$ of $T$. And $p$ is the unique solution in $F(T)$ to the following variational inequality:

$$
\langle(I-f) p, j(p-u)\rangle \leq 0
$$

for all $u \in F(T)$.

Lemma 3.7. Let $X$ be a reflexive Banach space which admits a weakly sequentially continuous duality mapping $J$ from $X$ to $X^{*}$. Suppose $C$ is a nonexpansive retract of $X$ which is also a sunny nonexpansive retract of $X$, and $T: C \rightarrow X$ is a nonexpansive mapping satisfying the weakly inward condition and $F(T) \neq \phi$, let $f: C \rightarrow C$ be a fixed contractive mapping from $C$ to $C$. And $\left\{x_{n}\right\}_{n=0}^{\infty}$ is a sequence by definition 2.4 (3). Let us assume that there are two sequences $\left\{\alpha_{n}\right\},\left\{\beta_{n}\right\}$ in $[0,1]$ satisfying the following conditions:

$$
\sum_{n=1}^{\infty}\left(\left|\alpha_{n}-\alpha_{n-1}\right|+\left|\beta_{n}-\beta_{n-1}\right|\right)<\infty
$$

then

1) $\lim _{n \rightarrow \infty}\left\|x_{n}-x_{n+1}\right\|=0$

2) $\lim _{n \rightarrow \infty}\left\|x_{n}-P T x_{n}\right\|=0$.

Proof by lemma 3.5, we know that the sequence $\left\{x_{n}\right\}$ is bounded. So the sequences $\left\{f\left(x_{n}\right)\right\},\left\{y_{n}\right\}, T x_{n}$ are also bounded. Therefore, we have:

$$
\begin{aligned}
\left\|y_{n}-y_{n-1}\right\| & =\left\|\beta_{n} x_{n}+\left(1-\beta_{n}\right) T x_{n}-\beta_{n-1} x_{n-1}-\left(1-\beta_{n-1}\right) T x_{n-1}\right\| \\
& =\left\|\beta_{n}\left(x_{n}-x_{n-1}\right)+\left(1-\beta_{n}\right)\left(T x_{n}-T x_{n-1}\right)+\left(\beta_{n}-\beta_{n-1}\right)\left(x_{n-1}-T x_{n-1}\right)\right\| \\
& \leq \beta_{n}\left\|x_{n}-x_{n-1}\right\|+\left(1-\beta_{n}\right)\left\|T x_{n}-T x_{n-1}\right\|+\mid \beta_{n}-\beta_{n-1}\left\|x_{n-1}-T x_{n-1}\right\| \\
& \leq\left\|x_{n}-x_{n-1}\right\|+\left|\beta_{n-1}-\beta_{n}\|\mid\| x_{n-1}-T x_{n-1} \|\right.
\end{aligned}
$$




$$
\begin{aligned}
\left\|x_{n+1}-x_{n}\right\| & =\left\|\alpha_{n} f\left(x_{n}\right)+\left(1-\alpha_{n}\right) P T y_{n}-\alpha_{n-1} f\left(x_{n-1}\right)-\left(1-\alpha_{n-1}\right) P T y_{n-1}\right\| \\
& =\left\|\alpha_{n}\left(f\left(x_{n}\right)-f\left(x_{n-1}\right)\right)+\left(\alpha_{n}-\alpha_{n-1}\right)\left(f\left(x_{n-1}\right)-P T y_{n-1}\right)+\left(1-\alpha_{n}\right)\left(P T y_{n}-P T y_{n-1}\right)\right\| \\
& =\alpha_{n} \beta\left\|x_{n}-x_{n-1}\right\|+\mid \alpha_{n}-\alpha_{n-1}\left\|f\left(x_{n-1}\right)-P T y_{n-1}\right\|+\left(1-\alpha_{n}\right)\left\|y_{n}-y_{n-1}\right\|
\end{aligned}
$$

by (8), we have

$$
\begin{aligned}
\left\|x_{n+1}-x_{n}\right\| \leq & \alpha_{n} \beta\left\|x_{n}-x_{n-1}\right\|+\left|\alpha_{n}-\alpha_{n-1}\right|\left\|f\left(x_{n-1}\right)-P T y_{n-1}\right\| \\
& +\left(1-\alpha_{n}\right)\left(\left\|x_{n}-x_{n-1}\right\|+\left|\beta_{n}-\beta_{n-1}\right|\left\|x_{n-1}-T x_{n-1}\right\|\right) \\
\leq & \alpha_{n} \beta\left\|x_{n}-x_{n-1}\right\|+\left|\alpha_{n}-\alpha_{n-1}\right|\left\|f\left(x_{n-1}\right)-P T y_{n-1}\right\| \\
& +\left(1-\alpha_{n}\right)\left\|x_{n}-x_{n-1}\right\|+\left|\beta_{n}-\beta_{n-1}\right|\left\|x_{n-1}-T x_{n-1}\right\| .
\end{aligned}
$$

Set $M_{2}=\max \left\{\left\|x_{n-1}-T x_{n-1}\right\|,\left\|f\left(x_{n-1}\right)-P T y_{n-1}\right\|\right\}$

$$
\begin{aligned}
\left\|x_{n+1}-x_{n}\right\| & \leq\left(1-\alpha_{n}+\alpha_{n} \beta\right)\left\|x_{n}-x_{n-1}\right\|+\left(\left|\alpha_{n-1}-\alpha_{n}\right|+\left|\beta_{n-1}-\beta_{n}\right|\right) M_{2} \\
& \leq\left(1-\alpha_{n}(1-\beta)\right)\left\|x_{n}-x_{n-1}\right\|+\left(\left|\alpha_{n-1}-\alpha_{n}\right|+\left|\beta_{n-1}-\beta_{n}\right|\right) M_{2} .
\end{aligned}
$$

Set $a_{n+1}=\left\|x_{n+1}-x_{n}\right\|, \quad t_{n}=1-\alpha_{n}(1-\beta), \quad b_{n}=0, \quad c_{n}=\left(\left|\alpha_{n-1}-\alpha_{n}\right|+\left|\beta_{n-1}-\beta_{n}\right|\right) M_{2}$

by the lemma 2.2 we have

$$
\lim _{n \rightarrow \infty}\left\|x_{n}-x_{n+1}\right\|=0
$$

Now we will proof $\left\|x_{n}-P T x_{n}\right\| \rightarrow 0$ as $n \rightarrow \infty$.

$$
\begin{aligned}
\left\|x_{n}-P T x_{n}\right\| & =\left\|x_{n}-x_{n+1}\right\|+\left\|x_{n+1}-P T x_{n}\right\| \\
& \leq\left\|x_{n}-x_{n+1}\right\|+\left\|\alpha_{n} f\left(x_{n}\right)+\left(1-\alpha_{n}\right) P T y_{n}-P T x_{n}\right\| \\
& \leq\left\|x_{n}-x_{n+1}\right\|+\alpha_{n}\left\|f\left(x_{n}\right)-P T x_{n}\right\|+\left(1-\alpha_{n}\right)\left\|P T y_{n}-P T x_{n}\right\| \\
& \leq\left\|x_{n}-x_{n+1}\right\|+\alpha_{n}\left\|f\left(x_{n}\right)-P T x_{n}\right\|+\left(1-\alpha_{n}\right)\left\|y_{n}-x_{n}\right\| \\
& \left\|y_{n}-x_{n}\right\|=\left\|\beta_{n} x_{n}+\left(1-\beta_{n}\right) T x_{n}-x_{n}\right\|=\left(1-\beta_{n}\right)\left\|x_{n}-T x_{n}\right\|
\end{aligned}
$$

as $n \rightarrow \infty, \alpha_{n} \rightarrow 0, \beta_{n} \rightarrow 1$ therefore

$$
\lim _{n \rightarrow \infty}\left\|x_{n}-P T x_{n}\right\|=0 .
$$

Remark 3.3. From the lemma 3.6 we know that $p$ is the unique solution in $F(T)$ to the following variational inequality:

$$
\langle(I-F) p, j(p-u)\rangle \leq 0 \text { for all } u \in F(T) .
$$

Now, we can take a subsequence $\left\{x_{n_{k}}\right\}$ of $\left\{x_{n}\right\}$ such that

$$
\limsup _{n \rightarrow \infty}\left\langle f(p)-p, j\left(x_{n}-p\right)\right\rangle=\limsup _{k \rightarrow \infty}\left\langle f(p)-p, j\left(x_{n_{k}}-p\right)\right\rangle
$$

we may assume that $x_{n_{k}} \rightarrow x^{*}$ by $X$ is reflexive and $\left\{x_{n}\right\}$ is bounded. It follows from Lemma 2.3, Lemma 2.4, and (10), we have $x^{*} \in F(T)=F(P T)$, by (11) we have

$$
\limsup _{n \rightarrow \infty}\left\langle f(p)-p, j\left(x_{n}-p\right)\right\rangle=\limsup _{k \rightarrow \infty}\left\langle f(p)-p, j\left(x_{n_{k}}-p\right)\right\rangle \leq 0 .
$$

Theorem 3.8. Let $X$ be a reflexive Banach space which admits a weakly sequentially continuous duality map- 
ping $J$ from $X$ to $X^{*}$. Suppose $C$ is a nonexpansive retract of $X$ which is also a sunny nonexpansive retract of $X$, and $T: C \rightarrow X$ is a nonexpansive mapping satisfying the weakly inward condition and $F(T) \neq \phi$, let $f: C \rightarrow C$ be a fixed contractive mapping from $C$ to $C$. And $\left\{x_{n}\right\}_{n=0}^{\infty}$ is the sequence by definition 2.4 (3). Let us assume there are two sequences $\left\{\alpha_{n}\right\},\left\{\beta_{n}\right\}$ in $[0,1]$ satisfying the following conditions:

$$
\sum_{n=1}^{\infty}\left(\left|\alpha_{n}-\alpha_{n-1}\right|+\left|\beta_{n}-\beta_{n-1}\right|\right)<\infty
$$

then the sequence $\left\{x_{n}\right\}$ converges strongly to the unique solution $p$ of the variational inequality:

$$
p \in F(T) \text { and }\langle(I-f) p, j(p-u)\rangle \leq 0 \text { for all } u \in F(T) .
$$

Proof. Since $C$ is closed, by lemma 3.5, $\left\{x_{n}\right\}$ is bounded, so $\left\{f\left(x_{n}\right)\right\},\left\{y_{n}\right\},\left\{T x_{n}\right\}$ are also bounded. Let $\left\{x_{t}\right\}$ be the sequence defined by

$$
x_{t}=P\left(t f\left(x_{t}\right)+(1-t) T x_{t}\right)
$$

by the lemma 3.6 as $t \rightarrow 0$ we have $\left\{x_{t}\right\}$ converges strongly to a fixed point $p$ of $T$ and $p$ is also the unique solution in $F(T)$ to the following variational inequality

$$
\langle(I-f) p, j(p-u)\rangle \leq 0 \text { for all } u \in F(T)
$$

using the remark 3.3, we have

$$
\limsup _{n \rightarrow \infty}\left\langle f(p)-p, j\left(x_{n}-p\right)\right\rangle \leq 0
$$

By the definition 2.4 (3), we have

$$
\begin{aligned}
\left\|x_{n+1}-p\right\|^{2}= & \left\|\alpha_{n} f\left(x_{n}\right)+\left(1-\alpha_{n}\right) P T y_{n}-p\right\|^{2} \\
\leq & \left\|\alpha_{n}\left(f\left(x_{n}\right)-p\right)+\left(1-\alpha_{n}\right)\left(P T y_{n}-p\right)\right\|^{2} \\
\leq & \left(1-\alpha_{n}\right)^{2}\left\|P T y_{n}-p\right\|^{2}+2 \alpha_{n}\left\langle f\left(x_{n}\right)-f(p), j\left(x_{n+1}-p\right)\right\rangle \\
& +2 \alpha_{n}\left\langle f(p)-p, j\left(x_{n+1}-p\right)\right\rangle \\
\leq & \left(1-\alpha_{n}\right)^{2}\left\|y_{n}-p\right\|^{2}+2 \alpha_{n} \beta\left\|x_{n}-p\right\|\left\|x_{n+1}-p\right\| \\
& +2 \alpha_{n}\left\langle f(p)-p, j\left(x_{n+1}-p\right)\right\rangle \\
\leq & \left(1-\alpha_{n}\right)^{2}\left\|y_{n}-p\right\|^{2}+\alpha_{n} \beta\left(\left\|x_{n}-p\right\|^{2}+\left\|x_{n+1}-p\right\|^{2}\right) \\
& +2 \alpha_{n}\left\langle f(p)-p, j\left(x_{n+1}-p\right)\right\rangle
\end{aligned}
$$

While

$$
\begin{aligned}
\left\|y_{n}-p\right\| & =\left\|\beta_{n} x_{n}+\left(1-\beta_{n}\right) T x_{n}-p\right\| \leq \beta_{n}\left\|x_{n}-p\right\|+\left(1-\beta_{n}\right)\left\|T x_{n}-p\right\| \\
& \leq \beta_{n}\left\|x_{n}-p\right\|+\left(1-\beta_{n}\right)\left\|x_{n}-p\right\|=\left\|x_{n}-p\right\|
\end{aligned}
$$

therefore,

$$
\begin{aligned}
\left\|x_{n+1}-p\right\|^{2} & \leq \frac{\left(1-\alpha_{n}\right)^{2}+\alpha_{n} \beta}{1-\alpha_{n} \beta}\left\|x_{n}-p\right\|^{2}+\frac{2 \alpha_{n}}{1-\alpha_{n} \beta}\left\langle f(p)-p, j\left(x_{n+1}-p\right)\right\rangle \\
& \leq\left(1-\frac{1-2 \alpha_{n} \beta}{1-\alpha_{n} \beta}\right)\left\|x_{n}-p\right\|^{2}+\frac{\left(1-\alpha_{n}\right)^{2}}{1-\alpha_{n} \beta}\left\|x_{n}-p\right\|^{2}+\frac{2 \alpha_{n}}{1-\alpha_{n} \beta} r_{n+1}
\end{aligned}
$$

where $r_{n+1}=\max \left\{\left\langle f(p)-p, j\left(x_{n+1}-p\right)\right\rangle, 0\right\}$ 
Setting $a_{n}=\left\|x_{n}-p\right\|, \quad t_{n}=1-\frac{1-2 \alpha_{n} \beta}{1-\alpha_{n} \beta}, \quad b_{n}=\frac{\left(1-\alpha_{n}\right)^{2}}{1-\alpha_{n} \beta}\left\|x_{n}-p\right\|^{2}, \quad c_{n}=\frac{2 \alpha_{n}}{1-\alpha_{n} \beta} r_{n+1} \quad$ and applying Lemma

2.1, we conclude that $x_{n} \rightarrow p$.

Let us prove $p$ is the unique fixed point of $T$.

We assume that $p^{*}$ is another solution of (12) in $F(T)$, then $\left\langle f(p)-p, j\left(p^{*}-p\right)\right\rangle \leq 0$ and $\left\langle f\left(p^{*}\right)-p^{*}, j\left(p-p^{*}\right)\right\rangle \leq 0$, so we have $(1-\alpha)\left\|p-p^{*}\right\| \leq 0$, which implies the equality $p=p^{*}$.

Remark 3.4. When $\beta_{n}=1$ for all $n \in \mathrm{N}$. The second type viscosity approximation methods for nonexpansive nonself-mappings (see definition 2.4) become the following iteration sequence:

$$
x_{n+1}=\alpha_{n} f\left(x_{n}\right)+\left(1-\alpha_{n}\right) P T x_{n} .
$$

So the theorem 3.8 improves the theorem 4.3 theorem 4.4 of Song-Li [2].

\section{Conclusion}

In this paper, we studied two new viscosity approximation methods for nonexpansive nonself-mappings, which were defined by definition 2.4. And then we proved that the sequences $\left\{x_{n}\right\}$ which were defined by definition 2.4 converged strongly to the fixed point of $T$, which were the nonexpansive nonself mappings in Banach space.

\section{References}

[1] Song, Y.S. and Chen, R.D. (2006) Viscosity Approximation Methods for Nonexpansive Nonself-Mappings. Journal of Mathematical Analysis and Applications, 321, 316-326.

[2] Song, Y.S. and Li, Q.C. (2007) Viscosity Approximation for Nonexpansive Nonself-Mappings in Reflexive Banach Space. J. Sys. Sci. and Math. Scis, 481-487. (In Chinese)

[3] Halpern, B. (1967) Fixed Points of Nonexpanding Maps. Bulletin of the American Mathematical Society, 73, $957-961$. http://dx.doi.org/10.1090/S0002-9904-1967-11864-0

[4] Kalinde, A.K. (1992) Fixed Points Ishikawa Iterations. Journal of Mathematical Analysis Applications, 600-606.

[5] Dotson, W.G. (1970) On the Mann Iterative Process. Transactions of the American Mathematical Society, 149, 65-73.

[6] Morales, C.H. and Jung, J.S. (2000) Convergence of Paths for Pseudo-Contractive Mappings in Banach Space. Proceedings of The American Mathematical Society, 128, 3411-3419. http://dx.doi.org/10.1090/S0002-9939-00-05573-8

[7] Liu, L.S. (1995) Ishikawa and Mann Iterative Processes with Errors for Nonlinear Strongly Accretive Mapping in Banach Spaces. Journal of Mathematical Analysis and Applications, 194, 114-125. http://dx.doi.org/10.1006/jmaa.1995.1289

\section{Submit or recommend next manuscript to SCIRP and we will provide best service for you:}

Accepting pre-submission inquiries through Email, Facebook, Linkedin, Twitter, etc A wide selection of journals (inclusive of 9 subjects, more than 200 journals) Providing a 24-hour high-quality service User-friendly online submission system Fair and swift peer-review system Efficient typesetting and proofreading procedure Display of the result of downloads and visits, as well as the number of cited articles Maximum dissemination of your research work

Submit your manuscript at: http://papersubmission.scirp.org/ 